University of Nebraska - Lincoln

DigitalCommons@University of Nebraska - Lincoln

Faculty Publications, Department of Psychology

Psychology, Department of

2009

\title{
Daily Negative Mood Affects Fasting Glucose in Type 2 Diabetes
}

\author{
Marilyn M. Skaff \\ University of California, San Francisco \\ Joseph T. Mullan \\ University of California, San Francisco \\ David M. Almeida \\ Pennsylvania State University \\ Lesa Hoffman \\ University of Nebraska-Lincoln, Ihoffman2@unl.edu \\ Umesh Masharani \\ University of California, San Francisco \\ See next page for additional authors
}

Follow this and additional works at: https://digitalcommons.unl.edu/psychfacpub

Part of the Psychiatry and Psychology Commons

Skaff, Marilyn M.; Mullan, Joseph T.; Almeida, David M.; Hoffman, Lesa; Masharani, Umesh; Mohr, David; and Fisher, Lawrence, "Daily Negative Mood Affects Fasting Glucose in Type 2 Diabetes" (2009). Faculty Publications, Department of Psychology. 375.

https://digitalcommons.unl.edu/psychfacpub/375

This Article is brought to you for free and open access by the Psychology, Department of at DigitalCommons@University of Nebraska - Lincoln. It has been accepted for inclusion in Faculty Publications, Department of Psychology by an authorized administrator of DigitalCommons@University of Nebraska - Lincoln. 


\section{Authors}

Marilyn M. Skaff, Joseph T. Mullan, David M. Almeida, Lesa Hoffman, Umesh Masharani, David Mohr, and Lawrence Fisher 
Published in Health Psychology 28:3 (2009), pp. 265-272; doi: 10.1037/a0014429 Copyright @ 2009 American Psychological Association. Used by permission. "This article may not exactly replicate the final version published in the APA journal. It is not the copy of record."

This research was supported by grants DK062732 and DK061937 from the National Institute of Diabetes, Digestive, and Kidney Disease. The following medical groups and diabetes education centers collaborated in this research: Alta Bates Diabetes Education Center, Brown and Toland Medical Group, CA Pacific Diabetes Education Center, Hill Physicians Medical Group, Marin IPA, St. Luke's Diabetes Education Center, St. Mary's Medical Center, University of California, San Francisco Hospital and Clinics. The authors thank Roche diagnostics for providing glucose monitors and strips.

\title{
Daily Negative Mood Affects Fasting Glucose in Type 2 Diabetes
}

\author{
Marilyn M. Skaff, Department of Family \& Medicine, University of California, San Francisco \\ Joseph T. Mullan, Department of Social \& Behavioral Science, University of California, San Francisco \\ David M. Almeida, Department of Human Development \& Family Studies, Pennsylvania State University \\ Lesa Hoffman, Department of Psychology, University of Nebraska-Lincoln \\ Umesh Masharani, Department of Medicine, University of California, San Francisco \\ David Mohr, Department of Preventive Medicine, Northwestern University \\ Lawrence Fisher, Department of Family \& Community Medicine, University of California, San Francisco \\ Corresponding author - Marilyn M. Skaff, Department of Family and Community Medicine, Box 0900, \\ University of California, San Francisco, 94143; email marilyn.skaff@ucsf.edu
}

\begin{abstract}
Objective: To examine the relationship between mood and blood glucose in a 21-day daily diary study.

Design: During a home visit, information was gathered from 206 persons with Type 2 diabetes regarding demographics, disease characteristics and treatment, and depressive symptoms. They had blood drawn at a laboratory, yielding HbA1C. The participants were then telephoned each evening for 21 days and were asked about their positive and negative mood during the past 24 hours. They also tested their blood glucose upon rising in the morning.

Main Outcome Measures: The main outcomes measures were positive and negative affect and fasting glucose.

Results: Multilevel analyses revealed a relationship between negative affect on one day and morning glucose on the next day. There was no such relationship between positive affect and glucose, nor was there a comparable effect of glucose on one day and either positive or negative affect on the next day.

Conclusion: The observed relationship between mood and blood glucose appears to be because of negative affect, not positive, with no evidence of a lagged effect of glucose on mood.
\end{abstract}

Keywords: negative affect, positive affect, diabetes, mood, glucose

It is estimated that people with diabetes have two to three times greater prevalence of depressive symptoms than those without diabetes (Anderson, Freedland, Clouse, \& Lustman, 2001; Lustman \& Clouse, 2005). For those persons living with diabetes, depression has potential negative consequences for diabetes complications, comorbidities, and mortality (de Groot, Anderson, Freedland, Clouse, \& Lustman, 2001). However, questions remain regarding the nature and direction of this relationship. Living with a chronic disease is a stressor that can negatively impact psychological well-being; yet, there is also increasing evidence that premorbid depression is a risk factor for the onset of Type 2 diabetes (Arroyo et al., 2004; Carnethon, Kinder, Fair, Stafford, \& Fortmann, 2003; Eaton, 2002; Everson-Rose et al., 2004; Palinkas, Lee, \& Barrett-Connor, 2004). Previous work on depression and diabetes has been largely cross-sectional and has inferred causal relationships by comparing across people or has looked at change, but usually over a relatively long period of time (months or years). This typical macroanalytic perspective links diagnostic categories of depression with global measures of glucose control such as $\mathrm{HbA1C}$. HbA1C represents a time weighted average blood glucose concentration over a 3-month period.

In this study, we asked how depressive affect and glucose may be related by adjusting our lens to focus on the day-today lives of persons living with diabetes. The study presented here takes a microanalytic view of mood and blood glucose, using daily diary methodology to examine positive and negative affect in relation to blood glucose over a 21-day period in the lives of persons with Type 2 diabetes.

\section{Positive and Negative Affect and Health}

Just as depression has been clearly shown to be related to diabetes and to health in general, there is a growing literature 
linking both positive and negative affect to health outcomes. Whereas in health literature, depression is more often viewed in terms of DSM-IV diagnostic categories and their clinical implications, it is likely as well that subclinical depressive symptoms, distress, mood, and affect may have implications for health outcomes and the general well-being of patients with diabetes. For example, Fisher and colleagues (2007) reported higher $\mathrm{HbA} 1 \mathrm{C}$ among those scoring above a cutoff of 16 and greater on the CES-D, compared to those $<16$, but no relationship between major depressive disorder (MDD), using a diagnostic interview, and $\mathrm{HbA1C}$.

Measures of affect usually differ from measures of depression not only in the time frame and persistence of specific feelings, but also in the breadth of emotions reflected in the measures. Many measures of affect include both positive affect (PA) and negative affect (NA), usually viewed as two distinct, orthogonal dimensions, rather than opposite ends of a bipolar dimension. The constructs of positive and negative affect refer more to what Denollet \& de Vries (2006) define as "subjective moods and feelings" (p. 172). They characterize positive affect as pleasant engagement with the environment, including such feelings as happiness, excitement, and contentment. Negative affect reflects distress and unpleasant reaction to the environment, involving such feelings as anger, fear, sadness, and nervousness.

Watson's Positive and Negative Affect Scale (PANAS) (Watson, Clark, \& Tellegen, 1988) was designed to measure orthogonal dimensions of positive and negative affect and has been widely used in studies of affect and health. When related, NA and PA are usually negatively correlated, although the strength of the correlation varies. Some of the variability in the relationship between the two is probably related to the time frame used as well as the intensity of the feelings (Pressman \& Cohen, 2005; Watson, Wiese, Vaidya, \& Tellegen, 1999). Daily measures of PA and NA tend to be less related to each other, partly because of the typically low to moderate levels of affect reported (Watson et al., 1999). The exception is in situations of high intensity, where an extreme in one would be incompatible with the other. When the time frame is longer, the correlations may be higher.

Depending on the time frame, affect can either reflect a relatively fleeting mood (state affect) or a more stable characteristic of the individual (trait affect). Generally, questions measuring state affect are framed "in the last day" or "currently," whereas trait affect is usually framed "in the last few weeks" or "generally" (Pressman \& Cohen, 2005). Of course, in daily studies as long as the present one (21 days), daily state affect measured repeatedly over several weeks might approach a typical or trait affect for the individual. A daily diary format allows many of the questions regarding characteristics of affect to be addressed in terms of how people differ in their average levels of PA and NA across the 21 days as well as how individuals vary day to day.

How are NA and PA related to depression? In most studies, affect and depressive symptoms are correlated. For example, Denollet and de Vries (2006) report a correlation of .58 between NA on the PANAS and depression as measured by the Centers for Epidemiologic Studies Depression scale (CES-D). Very similar results were reported by Watson et al. (1988) between the PANAS NA scale and the Beck Depression Inventory $(r=.57)$. Although there is far less information on positive affect, Watson et al. report correlations ranging from - 19 to -.36 between PA and depression symptom scales, depending on the depression scale and the time frame used. In a study of older persons that examined both daily affect and depression (CES-D), those who were categorized as having either "major depression" or "other depression" on the CES-D exhibited more NA and less PA (Chepenik et al., 2006). However, the authors report that even among those with major depression, there was a great deal of day-to-day variability in NA.

Similar to the models proposed for the relationship between depression and health (e.g., Cohen, Kessler, \& Gordon, 1995; Kiecolt-Glaser \& Glaser, 2002), the pathways between negative and positive affect and health outcomes may operate through behavioral and/or biological mediators, both of which have relevance for diabetes. Pressman and Cohen (2005) propose two models for the links between positive affect and health, although one might assume that such models would apply to negative affect as well. First, they suggest that affect has a direct effect on both behaviors and physiology. More specifically, they hypothesized that positive affect should result in better health behaviors and better adherence to treatment regimens. Direct physiological effects could include autonomic nervous system activation, HPA axis activation (decreased cortisol), and an effect on immune functioning (Kiecolt-Glaser, McGuire, Robles, \& Glaser, 2002; Taylor, Repetti, \& Seeman, 1997). Indeed, some evidence exists for a moderating effect of NA on the relationship between PA and natural killer cell activity (Valdimarsdottir \& Bougjerg, 1997).

In general, evidence is mixed regarding the effects of both positive and negative affect on health. Pressman and Cohen (2005) summarize the literature as showing that, in general, higher PA is related to lower mortality and morbidity, but point out that the data are often conflicting. Steptoe and Wardle (2005) found significant relationships for PA in the form of lower salivary cortisol, reduced fibrinogen stress response, and lower ambulatory heart rate (in men only) in an examination of data from the Whitehall study. Looking at NA in relationship to cardiovascular outcomes in a daily study, Kamarck, Schwartz, Shiffman, Muldoon, Sutton-Tirell, and Janicki (2005) differentiated between negative affect (valence) and arousal (activation) and found that NA was not related to heart rate changes, but arousal was.

There appears to be little evidence on the relationship between affect and blood glucose levels. In a study of adults with Type I and Type 2 diabetes, Trief, Himes, Orendorff, and Weinstock (2001) reported that high scores on the PANAS NA scale were related to more reported health problems and poorer marital quality. Although they found that marital quality was not significantly related to $\mathrm{HbA1C}$, they did not report the relationship between affect and glycemic control. Yancura and colleagues (Yancura, Aldwin, Levenson, \& Spiro, 2006) found no relationship between NA and fasting glucose in a study of metabolic syndrome, but PA was related to glucose. In a review article of research on affect and biological markers of health, Ryff et al. (2006) found evidence for a relationship between NA (but not PA) and glycemic control. In conclusion, the evidence is mixed for both PA and NA in their relationship to health, suggesting the need for a closer look at both affect and diabetes outcomes.

\section{Daily Diary Studies}

Daily (microanalytic) studies have increased in number in recent years because this type of methodology allows for both 
nomothetic (between person) and idiographic (within person) perspectives (Almeida, 2005; Tennen, Affleck, \& Armeli, 2005). Between person comparisons can tell us about how average levels of mood are related to average levels of glucose, whereas within-person analyses offer the opportunity to examine how intra individual variability in one variable (affect) is related to intra individual variability in another (glucose). For example, Charles and Almeida (2006) examined the relationship of affect with pain and respiratory and gastrointestinal (GI) symptoms in a daily diary study. They found both concurrent relationships and lagged relationships between negative affect and symptoms. All three types of symptoms were related to current state negative affect.

Although the number of daily studies has grown, there is little research to date examining daily variability in mood and glycemic control. Bode, Schwartz, Stubbs, and Block (2005) used continuous monitors over a 21-day period with participants with both Type I and Type 2 diabetes and reported considerable variability both within and between participants in blood glucose levels. The relationship between blood glucose variability and mood was examined in a daily study by Gonder-Frederick, Cox, Bobbitt, and Pennebaker (1989) involving 34 adults with Type 1 diabetes. Both positive and negative affect were measured four times each day, just before measuring blood glucose. In general, the authors reported that both positive and negative mood were related to blood glucose, although they described the relationships as "highly idiosyncratic," that is, the relationships varied across mood and among items used to measure mood.

In summary, the literature supports the relationship between mood/depression and diabetes outcomes, but the direction and strength of the relationship remains uncertain. Little evidence exists linking day-to-day mood and glucose. In the current study, we take a close-up view over 21 days of the relationship between mood and blood glucose. The benefits of this approach are that it allows us to tease out the mood/glucose relationships through multiple measures over time in a far more detailed manner than allowed in more macrolongitudinal research. Furthermore, it allows us to separate trait patterns from variations in mood state, observe the emergence of idiographic patterns, and test for moderators on the resulting patterns of relationship.

Adults with Type 2 diabetes participated in a 21-day daily study, in which they answered questions about positive and negative mood and tested their blood glucose. We asked, does negative or positive affect influence blood glucose and/or does blood glucose influence negative or positive affect over time? For both types of mood, there are two separate questions involving the between-person and withinperson elements of the predictors. Our primary questions include the following:

\section{Between persons:}

a. Are those higher in mean level of negative affect across the 21 days also higher in mean blood glucose?

b. Are those higher in mean level of positive affect across 21 days lower in mean blood glucose?

\section{Within person:}

a. Are those higher than usual in negative affect on one day higher than usual in fasting blood glucose on the following morning?

b. Are those higher than usual in positive affect on one day lower than usual in fasting blood glucose on the following morning?

c. Are those higher than usual in blood glucose on one day higher than usual in negative affect on the next day?

d. Are those higher than usual in blood glucose on one day lower than usual in positive affect on the next day?

In addition, we ask how stable between-person characteristics affect mean glucose levels and how they moderate the relationship between within-person affect and glucose.

\section{Method}

\section{Participants}

Participants for the daily study were drawn from a larger longitudinal study of depression, diabetes management, and glycemic control (Fisher et al., 2007). The longitudinal study participants were recruited from community-based Bay Area medical groups and diabetes centers. Inclusion criteria included a diagnosis of Type 2 diabetes, ages 21 to 75; Englishor Spanish-speaking; no severe diabetes complications or serious comorbidities.

Longitudinal study participants who entered that study before or during the recruitment period for the diary study were eligible for the diary study if they were English-speaking. Of the 452 persons who were eligible, 206 agreed to participate in the daily diary study $(50 \%)$. Participants in the diary study were paid $\$ 135$ (\$35 for the first week, $\$ 45$ for the second, and $\$ 55$ for the third week) for their participation. Demographic and disease related characteristics for both the diary sample and the 246 English-speaking nondiary participants in the longitudinal study are presented in Table 1 . The two samples did not differ significantly on any of these characteristics.

Table 1. Characteristics of Diary and Nondiary Participants (English Speaking)

\begin{tabular}{|c|c|c|c|c|c|}
\hline & \multicolumn{2}{|c|}{$\begin{array}{c}\text { Diary } \\
(N=206)\end{array}$} & \multicolumn{2}{|c|}{$\begin{array}{l}\text { Nondiary } \\
(N=246)\end{array}$} & \multirow[b]{2}{*}{$p$-level } \\
\hline & Mean & $(S D)$ & Mean & $(S D)$ & \\
\hline Female & $58.7 \%$ & & $54.5 \%$ & & 0.36 \\
\hline Age & 57.84 & $(9.13)$ & 57.74 & (10.53) & 0.92 \\
\hline Education (yrs) & 14.96 & $(2.70)$ & 15.41 & $(2.72)$ & 0.08 \\
\hline Ethnicity & & & & & 0.63 \\
\hline Asian & $20.4 \%$ & & $17.5 \%$ & & \\
\hline African-American & $22.8 \%$ & & $22.8 \%$ & & \\
\hline Latino & $10.2 \%$ & & $9.8 \%$ & & \\
\hline European-American & $37.9 \%$ & & $43.9 \%$ & & \\
\hline Other & $8.7 \%$ & & $6.1 \%$ & & \\
\hline Years since diagnosis & 8.00 & $(7.07)$ & 8.34 & (7.98) & 0.64 \\
\hline $\operatorname{HbA1C}(\%)$ & 7.22 & $(1.35)$ & 7.12 & $(1.32)$ & 0.42 \\
\hline Diabetes management & & & & & 0.26 \\
\hline Diet/exercise only & $13.6 \%$ & & $17.1 \%$ & & \\
\hline Oral meds only & $70.4 \%$ & & $68.7 \%$ & & \\
\hline Insulin only & $8.3 \%$ & & $4.5 \%$ & & \\
\hline Oral meds \& insulin & $7.8 \%$ & & $9.8 \%$ & & \\
\hline BMI & 33.19 & $(7.94)$ & 32.44 & $(7.74)$ & 0.31 \\
\hline CES-D & 10.49 & $(10.45)$ & 10.51 & $(10.26)$ & 0.98 \\
\hline Major Depression Dx & $8.3 \%$ & & $10.2 \%$ & & 0.49 \\
\hline
\end{tabular}

$p$-level based on $\chi^{2}$ or $t$ test. $N$ of cases varied slightly for those with numbers in last column: Educ, HbA1c, CESD, \& MDD. 
Baseline Interviews, Questionnaires, and Biological Measures

Participants completed questionnaires and were interviewed during a home visit lasting approximately 1.5 hours. During that visit, height and weight were measured from which body mass index (BMI) was calculated. In an interview, they were asked about the medications used to manage their diabetes and how long ago they had been diagnosed with diabetes (TDX). Diabetes medications were dummy coded into four categories (diet and exercise only, oral medications only, and both oral medications and insulin) with those on insulin only as the comparison group. They also visited a laboratory where a blood sample was collected, yielding hemoglobin A1C (HbA1C), a measure of glycemic control representing the percent of hemoglobin molecules circulating in the blood that contain sugar $(<7 \%$ is generally considered desirable for persons with Type 2 diabetes). A questionnaire asked about demographics including sex ( 1 = female), age, education (years completed), and self-identified ethnicity. Ethnicity was dummy coded into five categories (Asian, African American, Latino, Other) with Whites as the comparison group.

Depressive affect was measured with the Center for Epidemiological Studies Depression scale (CES-D) (Radloff, 1977), a 20 item measure asking about symptoms experienced in the past week (scores ranged from 0 to 60 ). The alpha for this sample was .84. Trained interviewers administered the Composite International Diagnostic Interview (CIDI) from which a diagnosis for Major Depressive Disorder (MDD) (Wittchen, 1994) was derived. For these analyses, a dichotomous variable was created where 1 indicates a diagnosis of MDD in the past year and 0 represents no MDD.

\section{Daily Diary Participants}

Participants ranged in age from 30 to 75, with a mean age of 57.8 (see Table 1). Slightly over half of the sample was female. The sample was ethnically diverse and relatively welleducated with approximately 3 years of college on average, although education ranged from 6 years or less to graduate degrees. They had been diagnosed with diabetes an average of 8 years and a majority $(70.4 \%)$ managed their disease with oral medications. The average HbA1C (7.22) indicates that they were managing their diabetes fairly well, although their average BMI of 33 is in the obese range. Only $8.3 \%$ meet criteria for MDD. Participation in the 21 days of the study was very high with $94.17 \%$ having complete data for at least 17 of the 21 days (98.5\% completed 14 or more days).

\section{Daily Diary Measures}

Each evening for 21 consecutive days, participants in the daily diary study received telephone calls from "diary recorders." Patients were run in groups ("flights") that were begun on the same night (always a week night). Nineteen flights consisting of 5 to 16 patients were run over 24 months. During the telephone calls, positive affect (PA) was measured by 10 items from the Positive Affect subscale of the Positive and Negative Affect Scale (PANAS; Watson, Clark, \& Tellegen, 1988), including interested, excited, strong, enthusiastic, proud, alert, inspired, determined, attentive, and active, as well as by four additional items of happy, pleased, content, and glad. Negative affect (NA) was measured by 10 items from the Negative Affect subscale of the PANAS, including distressed, upset, guilty, scared, hostile, irritable, ashamed, nervous, jittery, and afraid, as well as by five additional items: disappointed, sad, unhappy, troubled, and miserable. The new items were added to improve measurement precision across the range of each construct. For each item participants were asked to respond on a 5-point scale the extent to which they experienced that emotion within the past 24 hours, where $1=$ not at all, $2=$ a little, $3=$ moderately, $4=$ quite a bit, and $5=$ extremely.

Diary participants were given a Roche Accu-Check automated blood glucose monitor, with strips for 80 tests. They tested their blood glucose each morning upon arising (a.m. fasting glucose). Readings were stored in the monitor, along with the time they tested, and at the end of the 21 days, the research assistants returned to the participants' homes and downloaded the glucose readings.

\section{Item Response Analysis of Affect Items}

Confirmatory factor analyses were conducted to examine the extent to which the new affect items appeared to measure the same construct as the PANAS affect items. Item responses were modeled as categorical (i.e., as a graded response model) using the WLSMV estimator in Mplus 3.13 (Muthén \& Muthén, 1998-2004). Analyses were conducted on the 4087 daily observations with at least one response under the assumption of measurement invariance across days and missingness at random given other observed responses. Fit was acceptable. Further, all the factor loadings for the new items were above .75, comparable to standard measures, indicating that they did appear to measure the same latent construct as the PANAS items. Latent trait estimates $(M=0, S D=1)$ from separate single-factor models for positive and negative affect were then saved for further analysis. By using latent trait estimates rather than observed scores, measurement error is minimized from the daily responses and the noninterval nature of the response options is properly accounted for.

\section{Data Analysis Plan}

We conducted a multilevel analysis using SAS PROC MIXED to assess the effects of positive and negative mood on daily variations in morning blood glucose. We separated each measure of mood into two predictors, (a) a between-person measure representing the individual's mean across the 21 days [MnNA $_{\mathrm{i}}$ for Negative Affect and $\mathrm{MnPA}_{\mathrm{i}}$ for Positive Affect], and (b) a within-person measure representing the individual's deviation for the previous day from his or her 21-day mean $\left[\left(\operatorname{LagNA}_{\mathrm{di}}-\mathrm{MnNA}_{\mathrm{i}}\right)\right.$ for lagged Negative Affect], and [(Lag$\mathrm{PA}_{\mathrm{di}}-\mathrm{MnPA}_{\mathrm{i}}$ ) for lagged Positive Affect]. The between-person measures are centered around the grand mean by virtue of the latent variable constructed from the IRT. The within-person variables were centered about the person's mean: a higher or lower level represents a higher or lower difference on the previous day from the individual's mean.

This characterization of mood allows us to examine whether between-person or within-person differences in mood are related to blood glucose, for example, whether people with higher average levels of negative affect and positive affect have higher levels of fasting blood glucose and/or whether 
people's daily variation in negative affect and positive affect influences fasting blood glucose levels.

\section{Analytic Models}

A series of multilevel regression models were used to examine the relationship between logged morning glucose and negative and positive affect. Model A, as shown in Equation 1 , is the regression of morning glucose on negative affect and positive affect.

$$
\text { Level 1: } \quad \begin{aligned}
Y_{\mathrm{di}}=\beta_{0 \mathrm{i}}+\beta_{1 \mathrm{i}} & \left(\operatorname{LagNA}_{\mathrm{di}}-\mathrm{MnNA}_{\mathrm{i}}\right) \\
& +\beta_{2 \mathrm{i}}\left(\operatorname{LagPA}_{\mathrm{di}}-\text { MnPA }_{\mathrm{i}}\right)+\mathrm{r}_{\mathrm{di}}
\end{aligned}
$$

Level 2: $\quad \beta_{0 \mathrm{i}}=\gamma_{00}+\gamma_{01}\left(\mathrm{MnNA}_{\mathrm{i}}\right)+\gamma_{02}\left(\mathrm{MnPA}_{\mathrm{i}}\right)+\mathrm{U}_{0 \mathrm{i}}$

$$
\beta_{1 \mathrm{i}}=\gamma_{10}+\mathrm{U}_{1 \mathrm{i}}
$$$$
\beta_{2 \mathrm{i}}=\gamma_{20}+\mathrm{U}_{2 \mathrm{i}}
$$

In the Level 1 model, logged morning glucose $\left(\mathrm{Y}_{\mathrm{di}}\right)$ is a function of an individual intercept $\left(\beta_{0 \mathrm{i}}\right)$, the within-person effects of negative affect $\left(\beta_{1 \mathrm{i}}\right)$ and positive affect $\left(\beta_{2 \mathrm{i}}\right)$, and the residual morning glucose $\left(\mathrm{r}_{\mathrm{di}}\right)$ on day $d$ for individual $i$. In the Level 2 model, the individual intercept $\left(\beta_{0 \mathrm{i}}\right)$ is a function of a fixed intercept $\left(\gamma_{00}\right)$, the main effects of between-person positive and negative affect $\left(\gamma_{01}\right.$ and $\left.\gamma_{02}\right)$, and an individual-specific random intercept $\left(\mathrm{U}_{0 \mathrm{i}}\right)$. The individual effects of within-person negative affect $\left(\beta_{1 \mathrm{i}}\right)$ and positive affect $\left(\mathrm{B}_{2 \mathrm{i}}\right)$ are a function of the fixed effects $\left(\gamma_{10}\right.$ and $\left.\gamma_{20}\right)$ and individual-specific random effects of within-person negative and positive affect $\left(U_{1 \mathrm{i}}\right.$ and $\mathrm{U}_{2 \mathrm{i}}$ ). Because the previous day's negative affect was missing for the first day, only 20 days were included.

Model B adds a set of between-person variables to the Level 2 equations. We added variables in blocks to check for multicollinearity, to test for nonlinearity, and examine interactions with within-person variables. First we added background characteristics (gender, age, education, ethnicity, BMI, time with diabetes, and medicine regimen), then we added two indices of depression, MDD diagnosis in the past year and CESD symptoms. Finally, HbA1C level and its quadratic value were included. We explored a variety of covariance structures, a model with random effects for negative and positive affect, a first-order autoregressive model (AR1), a set of Toepliz models, and a model with heterogeneity associated with the between-person variables (Hoffman, in press; Littell, Milliken, Stroup, \& Wolfinger, 1996). For distinguishing between nested models with varying fixed effects, we used ML estimation; for nested models with varying random effects, we use REML estimation (Fitzmaurice, Laird, \& Ware, 2004).

\section{Results}

Table 2 presents between-person correlations for all variables. These between person correlations represent individuals' means across the 21 days. Gender was not related to any of the study variables, but older participants had lower CESD scores, had lower mean NA, had been diagnosed with diabetes longer than younger participants, and were more likely to be on insulin only. Those with higher education were less likely to be on insulin only and had lower scores on the CES$\mathrm{D}$, but education was not related to either positive or negative affect. Of particular interest is the relationship between PA and NA and their relationship to CES-D scores. There was a small, although significant, negative correlation between mean levels of NA and PA (-.16) and both were significantly related to the CES-D. CES-D scores were positively related to NA (.56) and negatively related to PA (-.24). Neither PA nor NA was significantly related to $\log \mathrm{HbA} 1 \mathrm{C}$.

ICCs, representing the proportion of variance between persons, indicate that significant variability exists both between and within persons: ICC for positive affect was $.71, .57$ for negative affect, and .69 for a.m. glucose. The within-person correlation between negative and positive affect was modest (-.20) and the within-person correlations were small, for example, the correlations of negative and positive affect with fasting glucose were .01 and .05 , respectively.

\section{Relationship of Lagged Affect on Morning Glucose}

Table 3 provides the parameter estimates for the fixed effects from Equation 1 and its extensions. The first column, Model A, provides the fixed effects of four variables, because

Table 2. Between Person Correlations: Pearson Correlation Coefficients

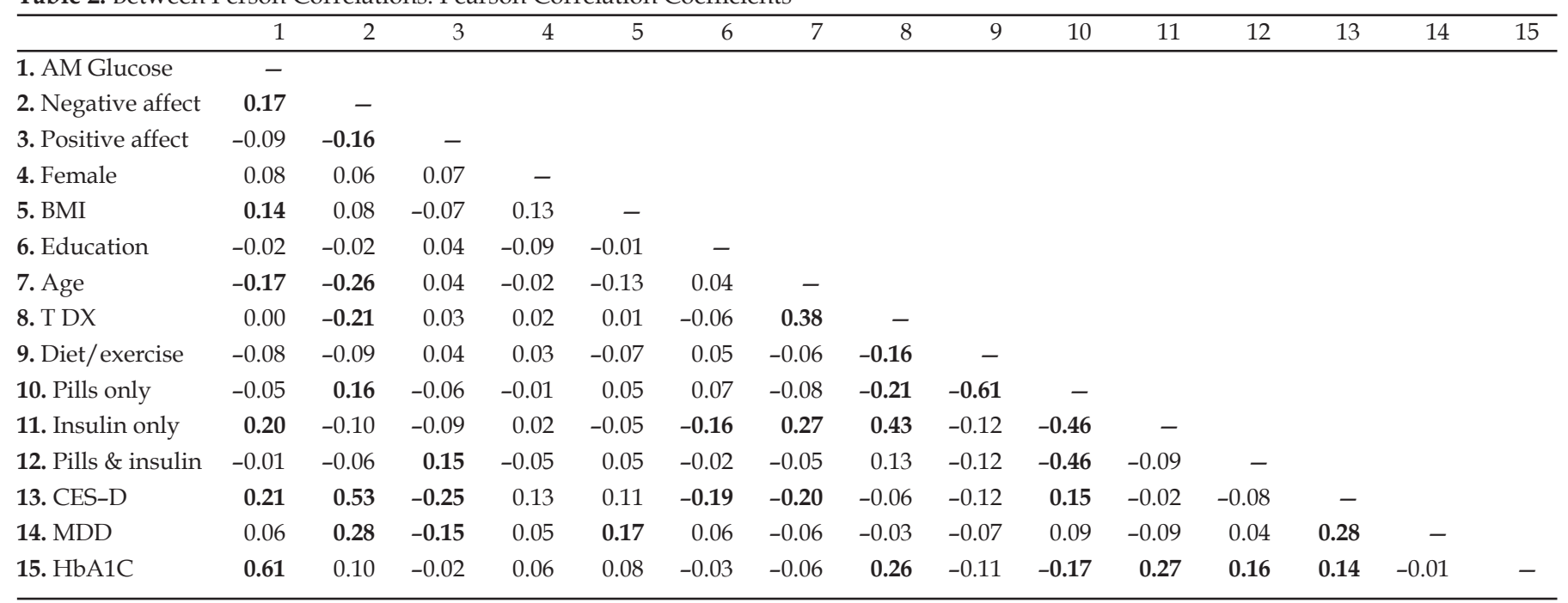

Correlations in bold are significant, at least at the 0.05 level. 
both negative and positive affect are each represented by two variables: the between-person variable (the average level across the 21 days) and the within-person variable (the deviation of the previous day's affect from their 21-day average). Conceptually, at Level 1, daily glucose is a function of an individual intercept and the within-person impact of negative and positive affect from the previous day. At Level 2, the individual intercept is a function of the fixed intercept and the main effects of between-person negative and positive affect (their averages over the 21 days).

There are significant coefficients for negative affect but not positive affect. The between-person coefficient indicates that persons higher in their overall level of negative affect across the 21 days have higher morning glucose levels, net of positive affect ("grumpy people" have higher glucose levels). The within-person coefficient, although modest, indicates that on days in which negative affect was higher than usual, the next morning's glucose was also higher than usual ("grumpy days" affect subsequent blood glucose levels). There was no evidence of collinearity at either level because of the modest correlation between negative and positive affect (within-person $r=-.20$; between-person $r=-.20$ ).

We also examined at each stage whether there were interactions between within-person and between-person effects. In Model A, we assessed whether levels of positive or negative affect moderated the relationship of prior within-person affect and subsequent blood glucose, asking if those with high average negative affect might be more susceptible to having daily variations in negative affect exert an influence on the next days blood glucose, or whether positive affect might lessen the impact of daily negative affect on blood glucose. No such interactions approached statistical significance.

Table 3. Predictors of Logged AM Glucose (Unstandardized Regression Weights With Standard Errors)

\begin{tabular}{lccccc}
\hline & \multicolumn{2}{c}{$\mathrm{A}$} & & \multicolumn{2}{c}{$\mathrm{B}$} \\
\cline { 2 - 3 } \cline { 6 - 6 } Effect & Estimate & $S E$ & & Estimate & \multicolumn{1}{c}{$S E$} \\
\hline Intercept & 4.9136 & $0.0195^{* * *}$ & 5.0692 & $0.0732^{* * *}$ \\
Lagged NA & 0.0152 & $0.0077^{*}$ & & 0.0123 & $0.0057^{*}$ \\
Mean NA & 0.0673 & $0.0315^{*}$ & -0.0148 & 0.0284 \\
Lagged PA & 0.0082 & 0.0073 & & 0.0023 & 0.0058 \\
Mean PA & -0.0198 & 0.0231 & -0.0165 & 0.0183 \\
Female & - & - & 0.0220 & 0.0298 \\
BMI & - & - & 0.0005 & 0.0021 \\
Education & - & - & 0.0042 & 0.0057 \\
Age & - & - & -0.0032 & 0.0018 \\
TDX & - & - & -0.0068 & $0.0026^{* *}$ \\
White vs. Asian & - & - & -0.0731 & 0.0428 \\
White vs. Black & - & - & -0.0464 & 0.0406 \\
White vs. Latino & - & - & -0.0127 & 0.0520 \\
White vs. Other & - & - & -0.0116 & 0.0562 \\
Insulin vs. Diet/Exer & - & - & -0.1665 & $0.0770^{*}$ \\
Insulin vs. Pills & - & - & -0.1493 & $0.0682^{*}$ \\
Insul. vs. Pills \& Insul. & - & - & -0.2535 & $0.0818^{* *}$ \\
CESD & - & - & 0.0003 & 0.0018 \\
MDDYR & - & - & 0.0035 & 0.0552 \\
HbA1C & - & - & 0.1602 & $0.0174^{* * *}$ \\
HbA1C sq & - & - & -0.0114 & $0.0053^{*}$ \\
\hline & & - & & &
\end{tabular}

${ }^{*} p<.05 ;{ }^{* *} p<.01 ;{ }^{* * *} p<.001$.
Model B adds a set of characteristics potentially related to average glucose levels and which could interact with daily positive and negative affect to influence blood glucose. These include standard background variables as well as two indicators of depression, number of recent symptoms (CES-D) and diagnosis in the past year (MDD), as well as HbA1C. The effect of average negative affect is no longer statistically significant. The magnitude of the within-person effect is similar to Model A and remains significant. In addition, time with diabetes, medicine regimen, and $\mathrm{HbA} 1 \mathrm{C}$ are significant predictors of blood glucose. As time with diabetes increases, there is a decrease in average blood glucose, net of HbA1C. Those using insulin injections alone had higher blood glucose levels than those using diet/exercise, just oral medications, and a combination of oral medications and insulin injections. As expected, higher levels of $\mathrm{HbA1C}$ are related to higher glucose up to a point, and then the effect levels off.

We examined a series of interactions to assess whether any of the between-person characteristics might moderate the effect of positive or negative affect on blood glucose. Only gender appeared to interact with negative affect. The within-person effect of negative affect appeared to occur for men (.031, $p$ $<.01)$ and not for women $(.002, n s)$. It is important to note that neither CES-D nor MDD is a significant predictor of blood glucose levels, nor was there any evidence that either moderates the affect-glucose relationship.

\section{Relationship of Glucose to Affect}

We also examined whether there was a comparable set of relationships between blood glucose and subsequent variations in negative and positive affect, that is, with prior day's blood glucose levels predicting negative and positive affect, with the same set of background variables, depression, and HbA1C. There is little evidence that average levels of blood glucose or daily variations in blood glucose affect negative or positive affect, nor that the relationship varied by moderators (other Level 2 variables) (tables are available from corresponding author).

\section{Discussion}

In this study, we focused in on the daily emotional ups and downs in a sample of participants with Type 2 diabetes, giving us the opportunity to examine the relationship between mood and a proximate health outcome: blood glucose. This outcome is important both because over time it can have implications for diabetes complications, comorbidities, and mortality and also because it is potentially sensitive to variability in mood. There has been little previous work looking at daily (state) affect and glucose levels, particularly over such an extended period of time in a large sample; therefore we set out to answer a set of questions about the relationship of both positive and negative affect to fasting glucose and the direction of the relationships.

\section{Between-Person Relationships}

The between-person relationship of negative affect and glucose disappeared once the between-person controls were entered into the multilevel analyses. Between person characteristics that remained statistically significant in the analysis 
predicting glucose included $\mathrm{HbA1C}$, insulin only, and time since diagnosis (negative). Because $\mathrm{HbA1C}$ is a standard measure of glycemic control, it would be expected to be related to average daily glucose. Time since diagnosis was positively related to blood glucose until we controlled for $\mathrm{HbA1C}$. The positive relationship between insulin use and higher levels of glucose may represent those having a more difficult time managing their disease.

\section{Within Person Negative Affect and Glucose}

The next questions we asked involved within-person analyses that allowed us to examine the lagged effect of both positive and negative affect on glucose and vice versa. The most salient, although modest, finding is the evidence for a lagged effect of negative affect on fasting glucose. That is, when individuals' levels of negative affect were higher than their own average level on one day, their fasting glucose was higher than their average on the next morning. In particular, it is notable that neither CESD nor MDD was related to glucose, nor did they moderate the effect of mood on glucose.

When possible moderators of the negative affect-glucose relationship were tested, the only one that was significant was gender. Thus, it appears that the effect of previous day's negative mood on morning glucose operated for men, but not for women. We can only speculate about why this was so. Although women usually show higher levels of depressive affect, there were no significant between person sex differences in any of the affective variables, including CES-D, MDD, negative affect, or positive affect, that might help explain our findings. Women tend to be more expressive of their emotions, whereas men tend to hold them inside (Nolen-Hoeksema \& Jackson, 2001), which might explain the physiological impact of NA. However, rumination also tends to be higher in women, which has been linked to cortisol levels (McCullough, Orsulak, Brandon, \& Akers, 2007) and cortisol, in turn, has been linked to fasting glucose in patients with Type 2 diabetes (Oltmanns et al., 2006). Because of the number of interactions tested, there is the possibility that one could be significant by chance, however, a recent publication reported a relationship between depression and artherosclerosis for men with Type 1 diabetes, but not for women (Spitzer et al., 2008). Clearly, this unexpected finding warrants further examination.

\section{Positive Affect}

There has been increasing evidence for a relationship between positive affect and health, yet our data do not support such a relationship. In the between person analysis, there was no relationship between mean levels of positive affect and fasting glucose. Likewise, within persons, there were no lagged effects of positive affect on next morning glucose. Some research has suggested a moderating effect of positive affect on the relationship between negative affect and health outcomes (Valdimarsdottir \& Bougjerg, 1997), but again our data do not support this. It may be temperament more than daily fluctuations in state positive mood that is operating in other studies that report an effect of positive mood on health. As reported from the ICCs, the proportion of between person variance was greater for positive affect than for negative affect (.71 vs. .57$)$, suggesting that positive affect may be more of a stable trait than negative affect. Although PA may have positive effects on health outcomes, our results are consistent with those reported by Yancura, Aldwin,
Levenson, and Spiro (2006), in which they found no relationship between PA and fasting glucose. Finally, it is possible that the psychoneuroimmunological concomitants of PA are different than those of NA and glucose may be less reactive to positive affect. That is, it could be that PA and NA affect different components of health differentially.

\section{Reconciling Macroanalytic and Microanalytic Perspectives}

In the introduction we discussed the evidence, seen from a macroanalytic view, that there is a relationship between depression and diabetes outcomes. How might the data presented here be related to that view? We have a correlation of .56 between CES-D and mean NA across the 21 days, closely resembling the relationships reported by a number of investigators between these two constructs (Denollet \& deVries, 2006; Watson et al., 1988) and a correlation of .61 between mean levels of fasting glucose and $\mathrm{HbA1C}$. Our results are consistent with the impact of mood on glucose, but do not provide support for the impact of glucose on mood. Perhaps our microanalytic view provides a glimpse of "allostatic load" in action (McEwen, 1998).

\section{Limitations and Conclusions}

Among the limitations of this paper are the possible links between diabetes and affect that we have not tested here. We can only speculate about possible mediating variables between negative mood and glucose; however, typical models suggest that there are behavioral, as well as endocrine and/or immunological mediators that could explain this relationship (Kiecolt-Glaser \& Glaser, 2002; Pressman \& Cohen, 2005; Steptoe \& Wardle, 2005; Valdimarsdottir \& Bougjerg, 1997). Unfortunately, we do not have data to test possible endocrine or immune mediators. A future examination will include health behaviors. In addition, although this is a relatively large and diverse sample, it is limited to those already being treated by clinics, thus limiting variability in depressive symptoms, glucose, and affect.

Future research in this area must move toward identifying the mediators in the relationship between negative mood and glucose. It also remains to describe the precursors of variability in mood such as personality traits and environmental stressors. Nevertheless, the evidence provided here of the lagged effect of negative mood on fasting glucose has implications clinically and for future investigations of the mechanisms linking daily experiences to glycemic control, with the potential for contributing to a better understanding of the relationship between depressive affect and health.

\section{References}

Almeida, D. M. (2005). Resilience and vulnerability to daily stressors assessed via diary methods. Current Directions in Psychological Science, 14, 64-68.

Anderson, R. J., Freedland, K. E., Clouse, R. E., \& Lustman, P. J. (2001). The prevalence of comorbid depression in adults with diabetes. Diabetes Care, 24, 1069-1078.

Arroyo, C., Hu, F. B., Ryan, L. M., Kawachi, I., Colditz, G. A., Speizer, F. E., et al. (2004). Depressive symptoms and risk of type 2 diabetes in women. Diabetes Care, 27, 129-133. 
Bode, B. W., Schwartz, S., Stubbs, H. A., \& Block, J. E. (2005). Glycemic characteristics in continuously monitored patients with type 1 and type 2 diabetes: Normative values. Diabetes Care, 28, 2361-2366.

Carnethon, M. R., Kinder, L. S., Fair, J. M., Stafford, R. S., \& Fortmann, S. P. (2003). Symptoms of depression as a risk factor for incident diabetes: Findings from the national health and nutrition examination epidemiologic follow-up study, 1971-1992. American Journal of Epidemiology, 158, 416-423.

Charles, S. T., \& Almeida, D. (2006). Daily reports of symptoms and negative affect: Not all symptoms are the same. Psychology $\mathcal{E}$ Health, 21, 1-17.

Chepenik, L. G., TenHave, T., Oslin, D., Datto, C., Zubritsky, C., \& Katz, I. R. (2006). A daily diary study of late life depression. American Journal of Geriatric Psychiatry, 14, 270-279.

Cohen, S., Kessler, R. C., \& Gordon, L. U. (1995). Strategies for measuring stress in studies of psychiatric and physical disorders. In S.Cohen, R. C.Kessler, \& L. U.Gordon (Eds.), Measuring stress: A guide for health and social scientists (pp. 3-26). New York: Oxford University Press.

de Groot, M., Anderson, R., Freedland, K. E., Clouse, R. E., \& Lustman, P. J. (2001). Association of depression and diabetes complications: A meta-analysis. Psychosomatic Medicine, 63, 619-630.

Denollet, J., \& deVries, J. (2006). Positive and negative affect within the realm of depression, stress, and fatigue: The two-factor distress model of the global mood scale (GMS). Journal of Affective Disorders, 91, 171-180.

Eaton, W. W. (2002). Epidemiologic evidence on the comorbidity of depression and diabetes. Journal of Psychosomatic Research, 53, 903-906.

Everson-Rose, S. A., Meyer, P. M., Powell, L. H., Pandey, D., Torrens, J. I., Kravity, H. M., et al. (2004). Depressive symptoms, insulin resistance, and risk of diabetes in women at midlife. Diabetes Care, 27, 2856-2862.

Fisher, L., Skaff, M. M., Mullan, J. T., Arean, P., Mohr, D., Masharani, U., et al. (2007). Clinical depression vs. Distress among patients with type 2 diabetes: Not just a question of semantics. Diabetes Care, 30, 542-548.

Fitzmaurice, G., Laird, N. M., \& Ware, J. H. (2004). Applied longitudinal analysis. New York: Wiley.

Gonder-Frederick, L. A., Cox, D. J., Bobbitt, S. A., \& Pennebaker, J. W. (1989). Mood changes associated with blood glucose fluctuations in insulin-dependent diabetes mellitus. Health Psychology, $8,45-59$.

Hoffman, L. (2007). Multilevel models for examining individual differences in within-person variation and covariation over time. Multivariate Behavioral Research, 42, 609-629.

Kamarck, T. W., Schwartz, J. E., Shiffman, S., Muldoon, M. F., Sutton-Tyrell, K., \& Janicki, D. L. (2005). Psychosocial stress and cardiovascular risk: What is the role of daily experience?Journal of Personality, 73, 1749-1774.

Kiecolt-Glaser, J. K., \& Glaser, R. (2002). Depression and immune function: Central pathways to morbidity and mortality. Journal of Psychosomatic Research, 53, 873-876.

Kiecolt-Glaser, J. K., McGuire, L., Robles, T. F., \& Glaser, R. (2002). Psychoneuroimmunology: Psychological influences on immune function and health. Journal of Consulting and Clinical Psychology, 70, 537-547.

Littell, R. C., Milliken, G. A., Stroup, W. W., \& Wolfinger, R. D. (1996). SAS system for mixed models. Cary, NC: SAS Institute.

Lustman, P. J., \& Clouse, R. E. (2005). Depression in diabetic pa- tients: The relationship between mood and glycemic control. Journal of Diabetes and its Complications, 19, 113-122.

McCullough, M. E., Orsulak, P., Brandon, A., \& Akers, L. (2007). Rumination, fear, and cortisol: An in vivo study of interpersonal transgressions. Health Psychology, 26, 126-132.

McEwen, B. S. (1998). Protective and damaging effects of stress mediators,Seminars in Medicine of the Beth Israel Deaconess Medical Center (pp. 171-179): Massachusetts Medical Society.

Muthén, B. O., \& Muthén, L. K. (1998-2004). Mplus user's guide (3rd ed.). Los Angeles: Muthén \& Muthén.

Nolen-Hoeksema, S., \& Jackson, B. (2001). Mediators of the gender difference in rumination. Psychology of Women, 25, 37-47.

Oltmanns, K. M., Dodt, B., Schultes, B., Raspr, H. H., Schweiger, U., Born, J., et al. (2006). Cortisol correlates with metabolic disturbances in a population study of type 2 diabetic patients. European Journal of Endocrinology, 154, 325-331.

Palinkas, L. A., Lee, P. P., \& Barrett-Connor, E. (2004). A prospective study of type 2 diabetes and depressive symptoms in the elderly: The Rancho Bernado study. Diabetes Medicine, 21, 1185-1191.

Pressman, S. D., \& Cohen, S. (2005). Does positive affect influence health? Psychological Bulletin, 131, 925-971.

Radloff, L. S. (1977). The CES-D scale: A self-report depression scale for research in the general population. Applied Psychological Measurement, 3, 385-401.

Ryff, C. D., Love, G. D., Urry, H. L., Muller, D., \& Rosenkranz, M. A. (2006). Psychological well-being and ill-being: Do they have distinct or mirrored biological correlates?Psychotherapy \& Psychosomatics, 75, 85-95.

Spitzer, C., Volzke, H., Barnow, S., Krohn, U., Wallaschofsskit, H., Ludemann, J., et al. (2008). Association between depression and subclinical carotid atherosclerosis in patients with type 1 diabetes. Diabetic Medicine, 25, 349-354.

Steptoe, A., \& Wardle, J. (2005). Positive affect and biological junction in everyday life. Neurobiology of Aging, 26S, S108-S112.

Taylor, S. E., Repetti, R. L., \& Seeman, T. (1997). Health psychology: What is an unhealthy environment and how does it get under the skin?Annual Review of Psychology, 48, 411-447.

Tennen, H., Affleck, G., \& Armeli, S. (2005). Personality and daily experience revisited. Journal of Personality, 73, 1465-1483.

Trief, P. M., Himes, C. L., Orendorff, R., \& Weinstock, R. S. (2001). The marital relationship and psychosocial adaptation and glycemic control of individuals with diabetes. Diabetes Care, 24, 1384-1389.

Valdimarsdottir, H. B., \& Bougjerg, D. H. (1997). Positive and negative mood: Association with natural killer cell activity. Psychology \& Health, 12, 319-327.

Watson, D. D., Clark, L. A., \& Tellegen, A. (1988). Development and validation of brief measures of positive and negative affect: The PANAS scales. Journal of Personality and Social Psychology, 54, 1063-1070.

Watson, D. D., Wiese, D., Vaidya, J., \& Tellegen, A. (1999). The two general activation systems of affect: Structural findings, evolutionary considerations, and psychobiological findings. Journal of Personality and Social Psychology, 76, 820-838.

Wittchen, H. U. (1994). Reliability and validity studies of the who: Composite intervational diagnostic interview (CIDI): A critical review. Journal of Psychiatric Research, 28, 57-84.

Yancura, L. A., Aldwin, C. M., Levenson, M. R., \& Spiro, A., III. (2006). Coping, affect, and the metabolic syndrome in older men: How does coping get under the skin?Journal of Gerontology, 61B, P295-P303. 